\title{
Stripes in Quantum Hall Double Layer Systems
}

\author{
L. Brey \\ Instituto de Ciencia de Materiales de Madrid, CSIC, 28049 Cantoblanco, Madrid, Spain. \\ H.A. Fertig \\ Department of Physics and Astronomy, University of Kentucky, Lexington, KY 40506-0055
}

(October 29, 2018)

We present results of a study of double layer quantum Hall systems in which each layer has a high-index Landau level that is half-filled. Hartree-Fock calculations indicate that, above a critical layer separation, the system becomes unstable to the formation of a unidirectional coherent charge density wave (UCCDW), which is related to stripe states in single layer systems. The UCCDW state supports a quantized Hall effect when there is tunneling between layers, and is always stable against formation of an isotropic Wigner crystal for Landau indices $N \geq 1$. The state does become unstable to the formation of modulations within the stripes at large enough layer separation. The UCCDW state supports low-energy modes associated with interlayer coherence. The coherence allows the formation of charged soliton excitations, which become gapless in the limit of vanishing tunneling. We argue that this may result in a novel "critical Hall state", characterized by a power law $I-V$ in tunneling experiments.

PACS number 73.40.Hm, 73.20Dx, 73.20Mf ]

\section{INTRODUCTION}

Recently, it has been established that quantum Hall systems in which several Landau levels are filled may support states with highly anisotropic transport properties [1]. High mobility two-dimensional electron systems, in perpendicular magnetic fields such that the filling factor $\nu$ is in the vicinity of $N+1 / 2$, with $N$ an odd integer greater than 7 [2], are found to have diagonal resistivity ratios $\rho_{x x} / \rho_{y y}$ as large as 3500 [3]. $\left(\nu \equiv N_{e} / N_{\phi}\right.$ is the ratio of the number of electrons to the orbital degeneracy of a Landau level $N_{\phi}=A B / \Phi_{0}$, where $B$ is the magnetic field, $A$ is the sample area and $\Phi_{0}$ is the magnetic flux quantum.) While a good deal of this anisotropy is purely geometric [4], the experiments clearly indicate a groundstate for the electrons of a highly anisotropic nature.

These results are very likely to be related to stripe phases that have been found in mean-field calculations [5,6] and exact diagonalization studies [7] of electrons with partially filled high Landau levels. When thermal and quantum fluctuations are included, it is useful to classify possible states of such systems according to their symmetries, which bear a close resemblance to those found in liquid crystals [8]. Of particular interest is the smectic state, which, in the presence of some (hitherto unidentified) orientational ordering field, should exhibit strong macroscopic transport anisotropies as observed in experiment. Quantum fluctuations in this state have been studied, with some models [9, 10] indicating the possibility of metallic behavior in the groundstate. (See, however, Ref. [11].) The mean-field stripe states are a natural first approximation to such smectic states.

It by now well appreciated that for smaller filling factors, the introduction of a second layer in the quantum Hall system (i.e., double quantum well systems) leads to new phenomena and a rich phase diagram 12]. For a partially filled lowest Landau level $(N=0)$, at total filling factor $\nu=1$ and for small layer separation $d$, a spontaneously broken symmetry can occur in which interlayer phase coherence develops in the absence of interlayer tunneling. In this state the system supports a Goldstone mode, which has a roton minimum at a wavevector $q \approx 1.2 \ell$. For separations $d>d_{c} \sim \ell$, this minimum goes to zero, indicating a phase transition [13] into a unidirectional, (interwell phase) coherent charge density wave (UCCDW). At still higher separations, there is another phase transition into a phase-coherent Wigner crystal state (CWC) with two non-collinear primitive lattice vectors 14 15 .

For $N=0$, these states with broken translational symmetry are usually preempted by strongly correlated Fermi-liquid states in which translational invariance is restored by quantum fluctuations [16]. However, in higher Landau levels the effective interaction among electrons in a single layer contains structure that favors such a mean field state relative to uniform correlated states [17, 18]. This makes the double layer electron system in higher Landau levels an excellent candidate for observation of the interesting translational broken-symmetry states described above.

In this article, we demonstrate within the Hartree-Fock approximation that such states do indeed occur for electrons systems with high filling factors. We will focus on systems with $\nu=2 \nu_{L}=2 \times(2 N+1 / 2)$, where $N=0,1,2, \ldots$ $N$ is the index of the highest occupied orbital Landau level (LL) and $\nu_{L}$ is the filling factor in each layer. Unlike the situation for single layers, we find circumstances under which the system supports stripe ordering and a quantized Hall conductance simultaneously. Physically, this arises due to the development of coherence between the edges of the individual stripes, which opens up a gap 
in the single particle spectrum. This coherence has interesting consequences for the collective modes of the system, as well as for its low-energy charged excitations.

The results of this work may be summarized as follows: i) For any value of $N$, and for small layer separation, the groundstate of this system has a uniform density and phase coherence between the layers. The system will support a quantized Hall conductance.

ii) In the limit where tunneling may be neglected, the energy spectrum of the system contains a gapless linear (Goldstone) mode due to the spontaneous interlayer coherence. This mode contains a roton minimum at wavevectors $q \sim 1 / \ell$, which vanishes when the layer separation $d$ exceeds some critical value $d_{c}$. The precise value of $d_{c}$ depends on $N$.

iii) For $d>d_{c}$, a state with unidirectional charge density wave order and phase coherence between the layers is lower in energy than the uniform density state. For $N \geq$ 1 and any value of the layer separation, the coherent unidirectional charge density wave has lower energy than the square or triangular Wigner crystal. For tunneling parameter $t \neq 0$, this state supports a quantized Hall effect.

iv) For small values of $t$, the linear regions in which the density has significant magnitudes in both layers - i.e., the regions between the centers of stripes in different layers - will support low energy collective modes. These excitations are analogous to spin waves of one-dimensional $X Y$ magnets in a magnetic field. They sustain soliton excitations which carry charge $\pm e$, whose energy scales as $\sqrt{t}$.

v) For $t=0$, the charged excitations become gapless and no quantized Hall effect can occur. At zero temperature the system may undergo a Kosterlitz-Thouless transition, in which, in the equivalent $1+1$ dimensional classical XY system [19], vortices unbind. The bound vortex state is very unusual, similar to quantum Hall states in having a vanishing tunneling conductance, but nevertheless being gapless and not supporting a plateau in the Hall conductivity. Because of the power law correlations inherent in systems with bound vortices, we call this a critical Hall state.

vi) At higher values of the layer separation the electron system undergoes a first order phase transition to a noncoherent unidirectional charge-density wave state with a weak longitudinal modulation of the charge density. This state also can be viewed as a highly anisotropic Wigner crystal (i.e., a stripe crystal.)

This paper is organized as follows. In Section II we describe the uniform coherent state and study its charge density excitations. From the softening of this excitation we obtain a phase diagram, in tunneling versus layer separation, for which the UCS becomes unstable. In Section III we discuss in more detail the method of calculation used for studying the translational broken symmetry ground states, and introduce the different phases analyzed. In Section IV we present our numerical results. Section $\mathrm{V}$ is devoted to a qualitative discussion of the ex- citations supported by the unidirectional coherent charge density wave state, and their consequences for quantum fluctuations. We conclude in Section VI with a summary.

\section{INSTABILITIES OF THE UNIFORM COHERENT STATE.}

In what follows, we consider our double layer system at total filling factor $\nu=4 N+1$. The $4 N$ part of this filling refers to the fully filled Landau levels, each of which has two spin states and two "layer states". In the large magnetic field limit, it is safe to assume these lower levels are filled and inert, and we do not explicitly include them in the calculations below. We also assume there is no spin texture [20] in the groundstate, which is always true if the Zeeman coupling is large. If the tunneling amplitude $t$ is smaller than the Zeeman coupling, then our results apply to filling factors $\nu=4 N+3$ mutatis mutandis.

For small separations between the layers, the groundstate of the system is expected to be a uniform coherent state, with wave function of the form

$$
\left|\Psi_{N}>=\prod_{X} \frac{1}{\sqrt{2}}\left(C_{N, X, l, \uparrow}^{+}+e^{i \theta} C_{N, X, r, \uparrow}^{+}\right)\right| N-1>
$$

where

$$
\left|N-1>=\prod_{X^{\prime} ; \sigma=\uparrow \downarrow ; N^{\prime}<N ; i=l, r} C_{N^{\prime}, X^{\prime}, i, \sigma}^{+}\right| 0>.
$$

In these equations $C_{N, X, l(r), \sigma}^{+}$creates an electron in the Landau level (LL) $N$, with guiding center orbital label $X$ and spin $\sigma$ in the left (right) well. $\mid 0>$ is the electron vacuum and $|N-1\rangle$ is the wavefunction which describes the system at filling factor $4 N$, for which all the states with LL orbital index $N^{\prime}<N$ are occupied. Note that since in Eq.(1) the product runs over all the possible values of $X$ this state corresponds to filling factor $\nu=4 N+1$. By construction this state has the same electronic charge in each well. Since we assume that the only active electron states are those in the highest LL with spin parallel to the field, in order to simplify the notation we drop the indices $N$ and $\uparrow$ in the creation and destruction operators in what follows.

The state described by Eq.(1) has long-range order in the phase difference between electrons in the two layers, $\theta$. In the absence of tunneling between layers, the energy is independent of $\theta$, and the system has a continuous broken symmetry. For $t \neq 0$, the ground state of the system is composed of a full LL of single-particle orbitals which are a symmetric combination of the left and right layer orbitals $(\theta=0)$. This wavefunction is exact for non-interacting electrons, and for $d \rightarrow 0$ we expect it to remain exact when interactions are included. For finite $d$, quantum fluctuations become important and the ground state described by Eq.(1) is only an approximation. Nevertheless, we expect 13] a broken symmetry ground state 
to survive for $d$ smaller that a critical value of the layer separation $d_{c}$.

In order to estimate $d_{c}$ we calculate the charge density excitations (CDE's) of the system. The CDE's are classified by a conserved wavevector $\mathbf{q}$ [21], and their dispersion can be obtained from the poles of the charge density response function. Neglecting mixing between LL's, it has the form

$\omega_{c d e}(q)=\left\{\left[\Delta^{H F}-V_{d}(q)\right]\left[\Delta^{H F}-V_{b}(q)+V_{a}(q)-V_{c}(q)\right]\right\}^{1 / 2}$,

where

$$
\begin{aligned}
\Delta^{H F} & =2 t+V_{d}(q=0), \\
V_{a}(q) & =\frac{e^{2}}{\epsilon \ell} \frac{1}{q \ell} V(q), \\
V_{b}(q) & =\frac{e^{2}}{\epsilon \ell} \int_{0}^{\infty} d\left(q^{\prime} \ell\right) J_{0}\left(q q^{\prime} \ell^{2}\right) V\left(q^{\prime}\right), \\
V_{c}(q) & =\frac{e^{2}}{\epsilon \ell} \frac{1}{q \ell} V(q) e^{-q d} \\
V_{d}(q) & =\frac{e^{2}}{\epsilon \ell} \int_{0}^{\infty} d\left(q^{\prime} \ell\right) J_{0}\left(q q^{\prime} \ell^{2}\right) V\left(q^{\prime}\right) e^{-q^{\prime} d}
\end{aligned}
$$

with

$$
V(q)=e^{-q^{2} \ell^{2} / 2}\left(L_{N}\left(q^{2} \ell^{2} / 2\right)\right)^{2},
$$

where $L_{N}(x)$ is a Laguerre polynomial. In these expressions $\ell=\sqrt{\hbar c / e B}$ is the magnetic length and $\epsilon$ is the dielectric constant of the host semiconductor. $V_{a}$ and $V_{b}$ are the direct and exchange intrawell Coulomb interaction, while $V_{c}$ and $V_{d}$ are the direct and exchange interwell Coulomb interactions [15].

We have computed $\omega_{\text {cde }}(q)$ for different values of $N$, and have plotted several representative curves (with $t=$ 0 ) in Fig. 1 for different values of $N$ and for a layer separation near $d_{c}$. For $t=0$, at small wavevector $q$ and any value of $N$, the dispersion curves increase linearly. This acoustic excitation is the Goldstone mode associated with the continuous broken symmetry; i.e., the spontaneous interlayer coherence. At intermediate $q$ the dispersion curves develop a dip which becomes soft at a wavevector $q_{c}$ for values of $d$ bigger than a critical distance $d_{c}(N)$. As discussed above, this indicates an instability of the state. We can see in Fig.1 that, in magnetic length units, the value of $d_{c}$ decreases as the LL index increases. At larger values of $q$ (not shown in Fig.1) the spectra corresponding to $N>0$ develop some structure associated with the zeros of the Laguerre polynomials appearing in the definition of $V(q)$.

Fig. 2 illustrates $d_{c}$ versus $t$ for a few values of $N$, and thus represents a phase diagram for the stability of the uniform coherent state (stable for small $d$ and large $t$ ) with respect to the formation of a density wave state (for large $d$ and small $t$ ). Note that the uniform state is stable for $N=2$ even at $t=0$. This is not the case for single layers, where at $\nu=1 / 2$ the system forms stripes. Evidently, the condensation energy for spontaneous interlayer coherence outweighs the gain in energy associated with stripe formation in the individual layers. For large enough $d$, however, the single layer tendency to form stripes must eventually become important. In order to more precisely characterize the ground state after the instability sets in, we study different broken translational symmetry states. This is the subject of the next Section.

\section{BROKEN TRANSLATIONAL SYMMETRY STATES}

In this Section we study different translational broken symmetry states of the electron gas confined in a DQW system. We adopt the Hartree-Fock approximation in the form introduced by Côté and MacDonald 22]; in this method the important quantities are the expectation values of the operators

$$
\rho_{j, j^{\prime}}(\mathbf{q})=\frac{1}{N_{\phi}} \sum_{X, X^{\prime}} e^{-i q_{x}\left(X+X^{\prime}\right) / 2} \delta_{X, X^{\prime}-q_{y} \ell^{2}} C_{X, j}^{+} C_{X^{\prime}, j^{\prime}} .
$$

Here the quantum numbers $j, j^{\prime}$ are layer indices. Details of the application of this method to bilayer systems may be found in Ref. [15].

The Hartree-Fock equations have a number of different solutions corresponding to different states of the electron gas in the DQW system. Each solution is characterized by a set of order parameters $\left\langle\rho_{i, j}(\mathbf{q})>\right.$; the ground state energy can be expressed solely in terms of these quantities. In the following calculations we consider a limited number of physically interesting solutions and compare their energies, and consider situations when transitions among these different states may occur.

\section{A. Uniform Coherent State (UCS).}

This state is described by Eq.(1); it is characterized for $\theta=0$ by $<\rho_{l, l}(0)>=<\rho_{r, r}(0)>=<\rho_{r, l}(0)>=<$ $\rho_{l, r}(0)>=1 / 2$, and all other order parameters zero. As discussed above, translational invariance is not broken in this phase, but there is interwell coherence which, at $t=$ 0 , breaks a $U(1)$ symmetry of the Hamiltonian. In Eq. 1, this arises because the energy of the state is independent of $\theta$ [13]. The UCS has always lower energy than the incoherent, $<\rho_{l, r}(0)>=0$, uniform state.

\section{B. Unidirectional Coherent CDW State (UCCDW)}

In this state $<\rho_{i, j}\left(n \mathbf{G}_{0}\right)>\neq 0, n=0, \pm 1, \pm 2, \ldots ;$ the translational symmetry in one direction is broken and interwell coherence is allowed. The value of $G_{0}$ is chosen 
to minimize the energy of the system. Furthermore, in order to have an energy minimum in this class of states, the CDW in the two wells must be shifted by a distance $\pi / G_{0}$ with respect to one another. For $d \rightarrow d_{c}$ the value of $G_{0}$ which minimizes the energy approaches $q_{c}$.

The UCCDW state may be written in the form

$$
\left|U C C D W>=\prod_{C}\left(\sqrt{\nu_{l}(X)} c_{X, l}^{+}+\sqrt{\nu_{r}(X)} e^{i \theta} c_{X, r}^{+}\right)\right| N-1>
$$

where $\nu_{l(r)}(X)$ is the occupation of the state $X$ in the left (right) well, and $\nu_{l}(X)+\nu_{r}(X)=1$. The local occupation factors $\nu_{i}(X)$ are periodic functions of $X$ with period $2 \pi / G_{0}$. With an appropriate choice of origin, the charge density is an even function of $X$, so the state may be constructed with only odd Fourier components of $<\rho_{i, i}\left(n G_{0}\right)>$. The local coherence between wells is given by the quantity $\sqrt{\nu_{l}(X) \nu_{r}(X)} e^{i \theta}$, which is even in $X$ and periodic with period $\pi / G_{0}$; thus $<\rho_{l, r}\left(n G_{0}\right)>\neq 0$ only when $n$ is even.

From the order parameters it is easy to obtain the band structure of the UCCDW phase. The eigenvalues depend on the guiding center coordinate $X$, and (up to an unimportant constant) are given by

$$
\epsilon(X)= \pm \sqrt{A(X)^{2}+B(X)^{2}}
$$

\section{Coherent WC state (CWC)}

In this state $\left.\left\{<\rho_{i, j}(\mathbf{G})\right\rangle\right\} \neq 0$, translational symmetry is broken along two non-collinear vectors, and interwell coherence is allowed. The reciprocal lattice vectors $\{\mathbf{G}\}$ define a two-dimensional lattice containing one electron per unit cell.

At intermediate values of $d$ the square lattice has lower energy than the triangular lattice, because the interstitial regions in a square lattice are larger than those of a triangular lattice, allowing a particularly low interwell Hartree energy [24]. At larger values of $d$ there will necessarily be a first order phase transition into a triangular lattice, since its intralayer Madelung energy is lower than that of the square lattice. However, because the Madelung energies of the two states are very close [25], this only occurs at a very large value of $d$. We thus do not further consider the triangular lattice for the purposes of this work; the Wigner crystals we discuss are square lattices, with unit cell lattice parameters $a_{x}=a_{y}=2 \sqrt{\pi} \ell$.

As in the case of the UCCDW, in order to minimize the interwell electrostatic energy, the lattice in the two wells are shifted by a vector $\left(a_{x} / 2, a_{y} / 2\right)$.

For systems modulated in two directions, such as the CWC phase, there are gaps in the excitation spectrum, but the densities at which they occur are not $B$ dependent and the Hall conductance is not expected to be quantized.

where

$$
A(X)=\sum_{n \neq 0} e^{i n G_{0} X}\left[V_{a}\left(n G_{0}\right)-V_{b}\left(n G_{0}\right)-V_{c}\left(n G_{0}\right)\right]<\rho_{i, i}\left(n G_{0}\right)>, \quad \text { D. Modulated Unidirectional CDW state. }
$$

$B(X)=-t-\sum_{n} V_{d}\left(n G_{0}\right)<\rho_{l, r}\left(n G_{0}\right)>$.

Since the total filling factor of the Nth Landau level is 1 , only the lowest energy band is occupied. In the UCCDW phase we define the gap, $\Delta_{U C C D W}$, as the difference in energy between the highest occupied state and the lowest empty state. In the coherent solution, $<\rho_{l, r}(0)>$ is always different from zero, so that $B(X) \neq 0$ for all $X$; in this case $\Delta_{U C C D W}>0$. It is important to recognize that the Hartree-Fock approximation overestimates the actual gap to create well-separated particlehole pairs above the UCCDW state, in particular because the groundstate band structure does not reflect the possible existence of soliton quasiparticles. As we discuss below, for $t>0$, a magnetic field-dependent gap is always present and a quantized Hall conductance will be observed [23]; for $t=0$ we shall that the quantized Hall effect is absent.

In the limit $d \rightarrow \infty, \nu_{l}(X) \nu_{r}(X)=0$ and the coherence between wells is lost; $\nu_{i}(X)$ can only be zero or one. This limit corresponds to two uncoupled 2DEG's each supporting an independent stripe state.
In this state the electron gas has modulations in the charge density along the direction of the stripes. Such modulations are known to significantly lower the energy of stripes in a single layer [26]. While the twodimensional long-range order that appears in such a state may not survive in the groundstate due to quantum fluctuations [9 11], the appearance of such modulations within Hartree-Fock reflects the tendency of the stripes to look like one-dimensional crystals at short length scales.

In the calculation we use either an oblique or a rectangular lattice with one electron per unit cell. In either case, the primitive lattice vectors have the form

$$
\begin{aligned}
& \mathbf{a}_{1}=\left(X_{0}, Z_{0}\right) \\
& \mathbf{a}_{2}=\left(0, Y_{0}\right),
\end{aligned}
$$

with $X_{0}=2 \pi / G_{0}$ and $Y_{0}=4 \pi \ell^{2} / X_{0}$. The parameter $Z_{0}=Y_{0} / 2$ for an oblique lattice, and $Z_{0}=0$ for a rectangular lattice. In single layer systems, it is found 26 that the energy is extremely insensitive to the parameter $Z_{0}$, but there is a very shallow minimum for $Z_{0}=Y_{0} / 2$. This turns out to be the case of the present system as well.

For the two layer system under consideration here, to minimize the interwell Madelung energy the charge in 
the two wells is shifted with respect to one another by a vector

$$
\left(\frac{a_{1}^{2}}{2 X_{0}}, 0\right) .
$$

This particular shift vector is the analog of what one finds in hexagonal close packed structures.

In this class of solutions we have never found a coherent state. Whenever $<\rho_{i, i}\left(G_{x}, G_{y}\right)>\neq 0$ for $G_{y} \neq 0$, we always find $<\rho_{l, r}(\mathbf{G})>=0$ for all set of $\mathbf{G}^{\prime} s$. Physically, this arises because formation of modulations competes with interwell coherence. The latter lowers the energy by introducing admixtures of single particle states near the Fermi surface in different wells. Modulations, by contrast, destroy the Fermi surface: modulated stripes essentially represent a highly anisotropic Wigner crystal state, with gaps everywhere in the band structure between occupied and unoccupied states. Thus, there are no low-energy states that may be admixed between wells. The MUCDW is a solution without coherence between the wells and we find that it is not possible to change continuously from the UCCDW phase to the MUCDW phase.

As in the CWC phase, the gap in the excitation spectrum which appears in the MUCDW phase is present over a range of densities. This phase does not exhibit a quantized Hall effect.

\section{RESULTS.}

We now present our numerical results for the states discussed in the last Section. In Fig.3 we plot the energy difference $E_{C W C}-E_{U C C D W}$ as a function of the layer separation for different values of $N$. A negative (positive) value of this quantity implies that the $\mathrm{CWC}$ has lower (higher) energy than the UCCDW state. Because the transition at $d_{c}$ is continuous, both energies, $E_{C W C}$ and $E_{U C C D W}$, tend to the energy of the UCS at $d=d_{c}$. For any value $d$ near but higher than $d_{c}(N)$, we find the UCCDW phase has lower energy than the CWC, indicating the instability at $d_{c}$ is a transition from the UCS to the UCCDW state. The driving energy for this transition comes from the difference between intra- and inter-well interactions, which increases with layer separation. For small $d$, interwell and intrawell interactions are rather similar; at larger layer separations lower energies can be attained by improving intrawell correlations via $<\rho_{i, i}\left(G_{0}\right)>$ taking on a non-zero value. This can be accomplished only by allowing the phase relationship between electrons in different wells to fluctuate, i.e., by lowering the expectation value of $<\rho_{l, r}(0)>$ (cf. Eq. 7).

For $N=0$ a second transition occurs from the UCCDW state to the CWC state at $d \sim 1.65 \ell$. As commented above in the $N=0$ case the fluctuations will probably melt this CWC state, forming two non-coherent highly correlated Fermi liquids $[16]$. For $N \geq 1$, the situation is different: there is no $\mathrm{UCCDW} \rightarrow \mathrm{CWC}$ transition.
We find that the UCCDW phase always has lower energy than the CWC state. The difference in energy between these states increases with $N$. Obviously this is closely related to the fact that for $N \geq 1$, in the $\mathrm{HF}$ approximation the stripe phase is lower in energy than the WC state [5, 6].

We now discuss the properties of the UCCDW state in more detail. For concreteness, we focus on the case $N=2, d=1.2 \ell$ and $t=0$. Most of the qualitative results, however, apply to all the UCCDW states for other parameters.

Fig. 4 illustrates the local filling factor in the left well $\nu_{l}(X)$ and the local coherence factor $\sqrt{\nu_{l}(X) \nu_{r}(X)}$ as a function of $X$. For this case $G_{0} \sim \ell^{-1}$, and the width of a unit cell is $\sim 2 \pi \ell$. Note that $\nu_{l}(X)$ is the particlehole conjugate of $\nu_{r}(X)$, i.e., $\nu_{r}(X)=1-\nu_{l}(X)$. The coherence between the wells is maximum in the region where the charge density is shared by both wells. Note that, as commented above, the interwell coherence order parameter has periodic $\pi / G_{0}$. For smaller values of $d$, the oscillations of the density between wells decreases until, at $d_{c}$, the charge density becomes uniform, with $\nu_{l}(X)=\nu_{r}(X)=1 / 2$. At this point the coherence factor becomes constant and takes on its maximum value of $1 / 2$. For larger values of the layer separation, $\nu_{l(r)}(X)$ changes more rapidly, and the coherence is significantly different from zero in thinner regions. As $d \rightarrow \infty$, the width of the coherent regions tends to zero, and the local occupation is just zero or unity.

In Fig. 5 we plot the band structure, Eq.(9), in the UCCDW case. For the parameters used in the calculations the Hartree-Fock results show a gap for the charged excitations. The gap is maximum in the regions where the charge is localized in one of the wells and minimum in the regions where the charge is shared by both wells. For small values of $d$, the band structure becomes flatter and the energy gap increases until, at $d_{c}$, the gap is constant in the Brillouin zone and takes its maximum value $2\left(t+V_{d}(0)\right)$. For larger values of $d$ the gap decreases, reaching its minimum value $2 t$ when $d \rightarrow \infty$. In the UCCDW phase, $<\rho_{l, r}(0)>\neq 0$, so that there is always a finite energy gap, although in some circumstances this gap is much smaller than the typical experimental temperatures. It should be noted that the spatial structure of this gap suggests that charged quasiparticles, either due to thermal fluctuations or to doping away from total filling factor $\nu=4 N+1$, will be much more mobile along the direction of the stripes than perpendicular to them. It would be most interesting if such anisotropy in double well transport could be observed experimentally.

It should be noted that in Eq. 6, the wavefunction for the UCCDW has a free parameter $\theta$, whose value for $t=0$ does not affect the energy. This implies that the UCCDW supports gapless modes (beyond phonons), due to the spontaneous coherence. The interesting aspect of this system is that, for large enough $d$, the coupling between the coherent regions will become negligible, and each linear region may support its own gapless mode. 
As discussed below, the resulting system is similar to a collection of one-dimensional $X Y$ ferromagnets. The fluctuations of this degree of freedom greatly reduces the charge gap for any value of $t$ compared to what is found in the band structure, and for $t=0$ we predict that such fluctuations will drive the gap to zero.

Returning to the Hartree-Fock results, the physical origin for the existence of a CDW in DQW systems is easy to understand. For $d$ near $d_{c}$ the Hartree-Fock one body potential has a minimum at a wavevector smaller than the reciprocal lattice vector associated with a $\mathrm{WC}$, and the electron gas can attain a lower energy by forming a UCCDW. As $d$ increases, the minimum in the effective potential moves to larger wavevectors. Because of this, for $N=0$ the WC becomes more stable than the unidirectional CDW. For $N \geq 1$, by contrast, the effective two-dimensional Coulomb interaction, $V(q)$, contains Laguerre polynomials that have zeros at decreasing wavevectors when $N$ increases. This produces a zero in the repulsive Hartree potential where the exchange interaction is strong, making the UCDW more stable than the WC at larger layer separations.

In Fig.6 we plot the order parameter $\left\langle\rho_{l, r}(0)>\right.$ and the energy gap $\Delta_{U C C D W}$ as a function of the layer separation. Note that in the UCCDW phase the coherence is non-zero even for large values of $d$. As $d$ increases the charge energy gap goes to its minimum value, but this is not so much due to the decrease in the interwell coherence as it is to the increase of the intrawell coherence $<\rho_{i, i}\left(G_{0}\right)>$.

Finally, we address the question of the value of the layer separation for which the two electron layers become decoupled, forming at $N>0$, a pair of noncoherent MUCDW states. To find this, one must compute the energies of the MUCDW and the UCCDW as functions of the layer separation. These may be found in Figs. 7 and 8 for the cases $N=2$ and $N=1$, respectively. As commented above, as a function of $d$, there is a crossing (first order phase transition) in energies between the MUCCDW and the UCCDW phases. The transitions occurs at $d \sim 1.6 \ell$ in the $N=2$ case and at $d \sim 1.4 \ell$ in the $N=1$ case, indicating that the UCDW phase becomes more stable as the LL index increases. From the value of the charged excitation gap, we expect that the QHE in double layer systems for $t \neq 0$ will disappear for large enough $d$ due to the UCCDW $\rightarrow$ MUCDW transition and not the UCS $\rightarrow$ MUCDW transition.

\section{COLLECTIVE MODES AND SOLITONS IN THE UCCDW STATE}

As mentioned above, the UCCDW state contains an interesting degree of freedom, the phase of the coherence factor in the regions where there is significant occupation of both the left and right wells (cf. Fig. 4). As in the case of the UCS, this phase may be thought of as a function of position, and from it low energy excitations may be constructed 12]. Interesting new physics emerges from this degree of freedom in the limit of large $d$, when the linear coherent regions become very narrow, and the magnitude of the coherence factor nearly vanished between these regions. In this situation we may view the coherent regions as a collection of one-dimensional systems which are, in a first approximation, uncoupled [27]. Each coherent region thus has its own phase, $\theta_{i}(y)$, where $i$ labels the individual coherent region, and we assume the stripes are parallel to the $\hat{y}$ direction.

Once we adopt a model in which the $\theta_{i}$ 's are uncoupled, the low energy statics and dynamics become identical to those of a domain wall between spins of opposite orientation in a filled Landau level. This system has been studied in Ref. [28], and we may use those results to understand what will happen in the double layer system. At long wavelengths, the energy functional for the phase takes the form

$$
E_{i}=\int d y\left[\frac{1}{2} \rho_{s}\left(\frac{\partial \theta_{i}}{\partial y}\right)^{2}-g t \cos \theta_{i}\right] .
$$

In the above equation, $\rho_{s}$ is a stiffness for the effective $X Y$ spin degree of freedom, and $g$ is analogous to a Landé $g$ factor, with $t$ playing the role of a magnetic field. The values of both $\rho_{s}$ and $g$ depend on the precise form the UCCDW wavefunction, and require a microscopic calculation to evaluate. Note both $\rho_{s}$ and $g$ remain finite in the limit $t \rightarrow 0$.

Eq. 9 has the form of a sine-Gordon model, and a number of interesting properties immediately follow. Of special importance is the fact that the system supports soliton excitations, where $\theta_{i}$ changes by $2 \pi$ in $y$ going from $-\infty$ to $\infty$ [29]. The energy of these excitations have the form $\varepsilon_{s}=8 \sqrt{\rho_{s} g t}$. For reasons very similar to what occurs in double layer quantum Hall systems near $\nu=1$ [12], or analogously for systems with spin [30], the sine-Gordon model solitons carry a net charge of $\pm e$ [28]. This means the linear coherent regions support charged excitations much lower in energy than predicted by the Hartree-Fock theory for small $t$ : as discussed above, the energy to create a well-separated particle-hole pair tends to a constant as $t \rightarrow 0$, while the soliton energy vanishes as $\sqrt{t}$. For small $t$, at finite temperature the solitons should dominate the transport properties of the system.

In the limit of vanishing tunneling amplitude, a very interesting possibility arises for behavior of the linear coherent regions. As stated above, the system will have gapless modes associated with the spontaneously broken symmetry; these lead [28] to a set of collective modes dispersing linearly: $\omega(q)=A q$ 31. The action consistent with this has the form

$$
S=\int d \tau d y\left[\frac{1}{2} \rho_{\tau}\left(\frac{\partial \theta_{i}}{\partial \tau}\right)^{2}+\frac{1}{2} \rho_{s}\left(\frac{\partial \theta_{i}}{\partial y}\right)^{2}\right],
$$

where $\tau$ is imaginary time, and $\rho_{\tau}$ is an effective moment of inertia that depends on the precise form of the UC- 
CDW wavefunction. At zero temperature, Eq. 10 may be reinterpreted as an energy functional in a $1+1$ dimensional classical system 19. This system will undergo a Kosterlitz-Thouless transition if $\sqrt{\rho_{s} \rho_{\tau}}$ passes through $2 / \pi$. Above this value, vortices in the $\theta_{i}$ field are bound in pairs; below it, they are unbound.

The meaning of this phase transition may be understood by examining what happens for small but nonvanishing $t$. In this case, vortices are bound by a linear potential, and there will be a "string" attaching them in which $\theta_{i}$ rotates by $2 \pi \sqrt{12}$. bFrom the spin-charge coupling inherent in such multicomponent systems, this means the vortices "cap" a region in spacetime in which the total charge in the $i-t h$ linear coherent region fluctuates away from the average by $\pm e$. For $t>0$ the vortices always remain bound, and these fluctuations are controlled. Thus, fluctuations in the charge density of the 2DEG are suppressed, and the system exhibits a quantized Hall conductance.

For $t=0$ and $\sqrt{\rho_{s} \rho_{\tau}}>2 / \pi$, the vortices also remain bound, albeit logarithmically. This suggests that the charge density fluctuations are still suppressed, and the system bears a resemblance to a quantum Hall phase. However, since charged excitations are not gapped at $t=0$, the Hall conductance should not exhibit a plateau. The difference between states with bound and unbound vortices would be most directly probed by a tunneling experiment: in the bound-vortex phase, one expects a tunneling current $I$ that vanishes as a power of the voltage $V$ that is greater than one, so that the tunneling conductance vanishes. In the unbound state, the presence of free vortices means charge may be injected into the system, resulting in a finite tunneling conductance. Note that for a quantized Hall state, the tunneling current should vanish as $I \sim e^{-V_{0} / V}$, where $V_{0}$ is proportional to the quasiparticle gap [32]. The differing behaviors of the low-voltage $I-V$ characteristics indicates that the bound vortex state is qualitatively different than either a conducting state or a quantum Hall state. We tentatively call this new state a critical Hall state.

In closing this Section, we note that in real systems, the true groundstate of the DQW can never be a critical Hall state, because the tunneling amplitude can never be made to completely vanish in practice. In addition, there may be small but relevant gradient couplings among the different linear coherent regions, driving the system away from the critical Hall state at low enough temperature. In practice, both these perturbations may be made very small, so that at experimentally realizable temperatures their effects might be unimportant. It is important to evaluate whether the system parameters (i.e., $t, N$ and $d$ ) can be adjusted so that properties of a critical Hall state may be observed in practice. This will be the subject of a future study.

\section{SUMMARY}

In this paper, we studied double layer quantum Hall systems in which each layer has a high-index Landau level that is half-filled. Hartree-Fock calculations revealed that for small layer separations, the groundstate is a uniform coherent state (UCS). Above a critical layer separation $d_{c}(N)$, we found a continuous phase transition to a unidirectional coherent charge density wave (UCCDW), which is related to stripe states in single layer systems. This UCCDW state supports a quantized Hall effect when there is tunneling between layers $(t \neq 0)$, and is always stable against formation of a coherent Wigner crystal (CWC) for Landau indices $N \geq 1$. The state does become unstable to the formation of a modulated unidirectional charge density wave (MUCDW) state for large enough layer separation, which should lead to the destruction of the quantum Hall effect.

The UCCDW state supports interesting low-energy modes associated with interlayer coherence, which become gapless in the limit $t \rightarrow 0$. For relatively large layer separations, in a model where the Coulomb interaction is screened, the regions of coherence may be treated as independent one-dimensional systems with an $X Y$ degree of freedom. The resulting effective Hamiltonian is a sineGordon model, which supports charge soliton excitations, whose energy vanishes in the zero tunneling limit. At zero temperature, the equivalent $1+1$ dimensional system may be in a state with either bound or unbound vortices. Finally, we argued that the former possibility is an unusual situation which we call a "critical Hall state", and is characterized by a power law tunneling $I-V$ characteristic.

We thank A.H.MacDonald, C.Tejedor, L.MartínMoreno and J.J.Palacios for useful discussions. This work was supported by the CICyT of Spain under Contract No. PB96-0085, and by the NSF under Grant No. DMR98-70681.

[1] M.P.Lilly, K.B.Cooper, J.P.Eisenstein, L.N.Pfeiffer and K.W.West, Phys.Rev.Lett. 82, 394 (1999); R.R.Du, D.C.Tsui, H.L.Stormer, L.N.Pfeiffer, K.W.Baldwin and K.W.west, Solid State Comm. 109, 389 (1999).

[2] Anisotropic behavior may also be induced for $N=1$ in tilted samples. See Ref. [3.

[3] M.P. Lilly, K.B. Cooper, J.P. Eisenstein, L.N. Pfeiffer, and K.W. West, Phys. Rev. Lett. 83, 824 (1999).

[4] S.H. Simon, cond-mat/9903086.

[5] A.A.Koulakov, M.M.Fogler and B.I.Shklovskii, Phys.Rev.Lett. 76, 499 (1996).

[6] R.Moessner and J.T.Chalker, Phys.Rev.B 54, 50006 (1996). 
[7] E.H.Rezayi, F.D.M.Haldane and Kun Yang condmat/9903258.

[8] E. Fradkin and S.A. Kivelson, Phys.Rev.B 59, 8065 (1999).

[9] H.A.Fertig, Phys.Rev.Lett. 82, 3693 (1999).

[10] V. J. Emery, E. Fradkin, and S.A. Kivelson, condmat/0001077.

[11] A.H.MacDonald and M.P.A.Fisher, cond-mat/9907278.

[12] For a review, see article by S.M. Girvin and A.H. Macdonald in Perspectives in Quantum Hall Effects, (Wiley, New York, 1997).

[13] H.A.Fertig

Phys.Rev.B

40, 1087 (1989); A.H.MacDonald, P.M.Platzman, and G.S.Boebinger, Phys.Rev.Lett. 65, 775 (1990); L.Brey, Phys.Rev.Lett. 65, 903 (1990); X.M.Chen and J.J.Quinn, Phys.Rev.Lett. 67, 895 (1991); X.G.Wen and A.Zee, Phys.Rev.Lett. 69, 1811 (1992); Z.Ezawa and A.Iwazaki, J.Mod.Phys. B 19, 3205 (1992).

[14] X.M.Chen and J.J.Quinn, Phys.Rev.B 45, 11054 (1992).

[15] R.Côté, L.Brey and A.H.MacDonald, Phys.Rev.B 46, 10239 (1992).

[16] B.I.Halperin, P.A.Lee, and N.Read, Phys.Rev.B 47, 7312 (1993).

[17] M.M. Fogler and A.A. Koulakov, Phys. Rev. B 55, 9326 (1997).

[18] T.Jungwirth, A.H.MacDonald, L.Smrcka and S.M.Girvin, cond-mat/9905353; T. Stanescu, I. Martin, and P. Phillips, cond-mat/9905116.

[19] S.L. Sondhi, S.M. Girvin, J.P. Carini, and D. Shahar, Rev. Mod. Phys. 69, 315 (1997).

[20] H.A. Fertig, L. Brey, R. Côté, and A.H. MacDonald, Phys. Rev. B 50, 11018 (1994).

[21] C.Kallin and B.I.Halperin, Phys.Rev.B 30, 5655 (1984).

[22] R.Côté and A.H.MacDonald, Phys.Rev.Lett. 65, 2662 (1990); Phys.Rev.B 44, 8759 (1991).

[23] See, for example A.H.MacDonald in Proceedings of the Les Houches Summer School on Mesoscopic Physics (North-Holland, Amsterdam, 1995).

[24] L. Zheng and H.A. Fertig, Phys. Rev. B 52, 12282 (1995).

[25] L. Bonsall and A.A. Maradudin, Phys. Rev. B 15, 1959 (1977).

[26] R. Côté and H.A. Fertig, cond-mat/0001169.

[27] In many situations, the regions will be coupled by the long-range Coulomb interaction. For the purposes of this discussion, we ignore this coupling. This may be accomplished by the introduction of a screening plane parallel to the layers.

[28] V.I. Fal'ko and S.V. Iordanskii, Phys. Rev. Lett. 82, 402 (1999).

[29] R. Rajaraman, Solitons and Instantons, (North-Holland, New York, 1989).

[30] D.H. Lee and C.L. Kane, Phys. Rev. Lett. 64, 1313 (1990); S.L. Sondhi, A. Karlhede, S.A. Kivelson, and E.H. Rezayi, Phys. Rev. B 47, 16419 (1993).

[31] When Coulomb interactions between the linear coherent regions are included, the coefficient $A$ is a function of $q_{\perp}$, the wavevector describing the relative phases of the Goldstone excitations in each region.

[32] Song He, P.M. Platzman, and B.I. Halperin, Phys. Rev. Lett. 71, 777 (1993).

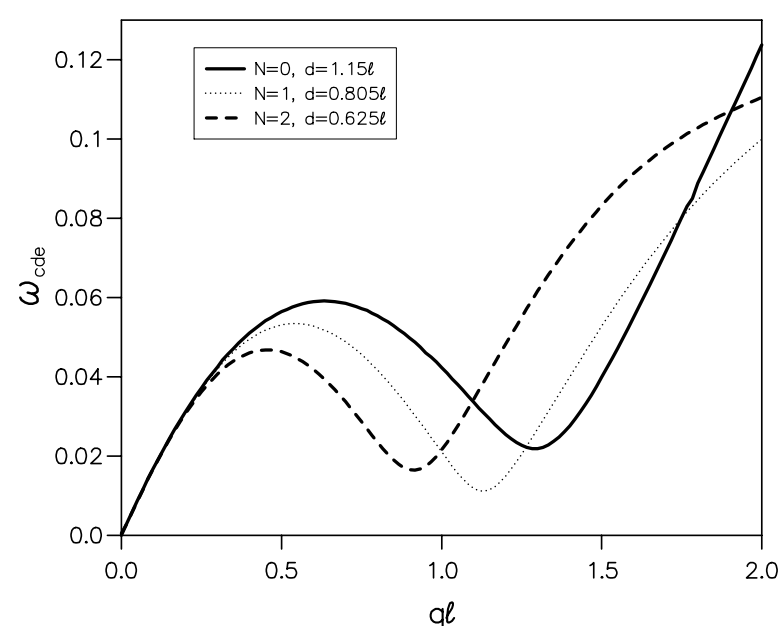

FIG. 1. Dispersion of the charge density excitations as a function of the wavevector $q$ for a double quantum well at filling factor $\nu=4 N+1$, and $t=0$. The value of the layer separation is chosen to be near the critical distance where the charge density instability occurs.

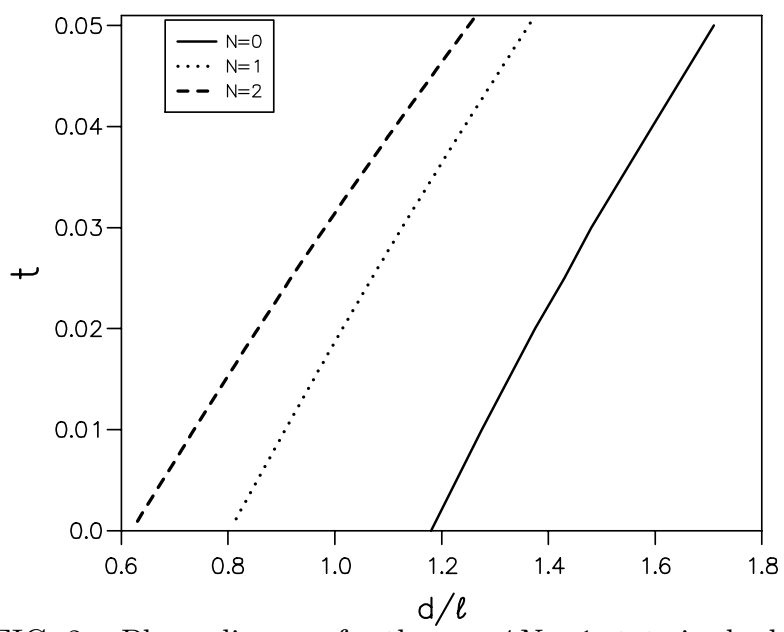

FIG. 2. Phase diagram for the $\nu=4 N+1$ state in double quantum well system. The line separates the region where the uniform coherent state occurs (small $\mathrm{d}$ and large $t$ ) from the region where a translational broken symmetry ground state is expected (large $d$ and small $t$ ). 


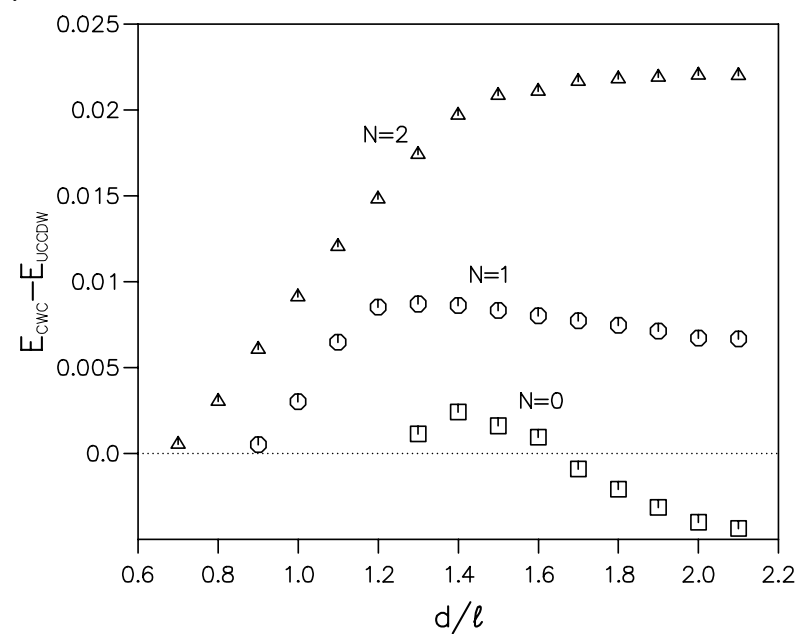

FIG. 3. Difference in energy between the CWC and the UCCDW states as a function of $d$. A positive(negative) value of this quantity means that the UCCDW state has lower (higher) energy than the CWC solution.

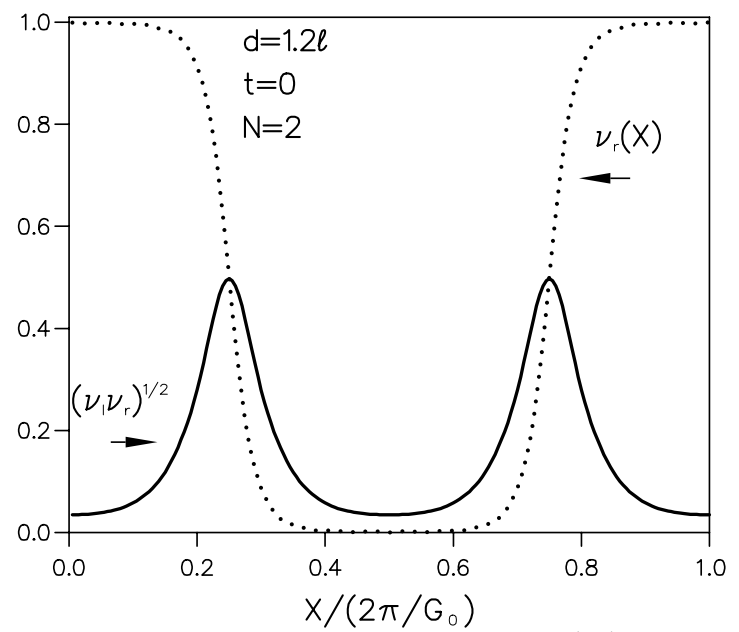

FIG. 4. Local filling factor in the left well $\nu_{l}(X)$ and local coherence $\sqrt{\nu_{l}(X) \nu_{r}(X)}$ as a function of $X$. These results correspond to $N=2, t=0$ and $d=1.2 \ell$. The length of the unit cell is $2 \pi / G_{0}$, and for this case $G_{0} \ell \sim 1$.

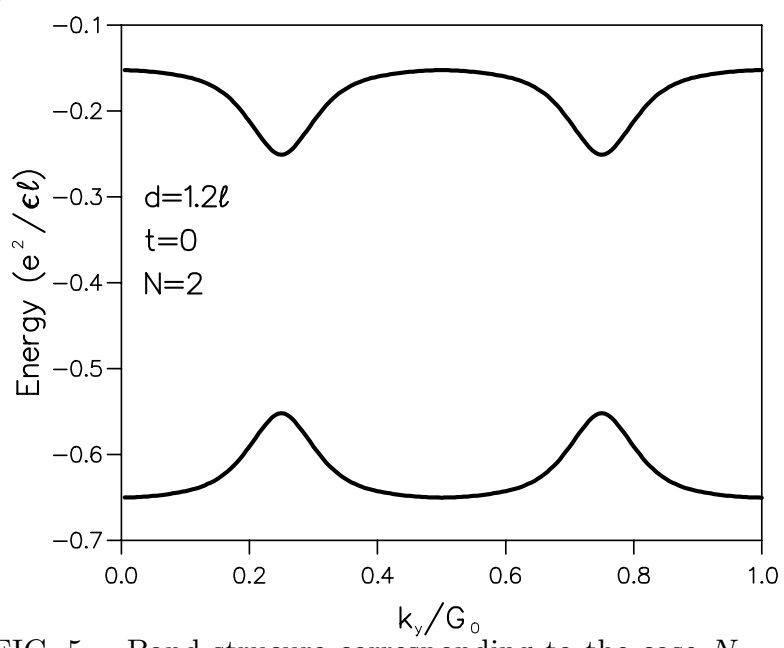

FIG. 5. Band strucure corresponding to the case $N=2$, $d=1.2 \ell$ and $t=0$. The eigenvalues depend on the momentum alon the $y$ direction, $X / \ell^{2}$, which is defined in the first Brilluin zone.

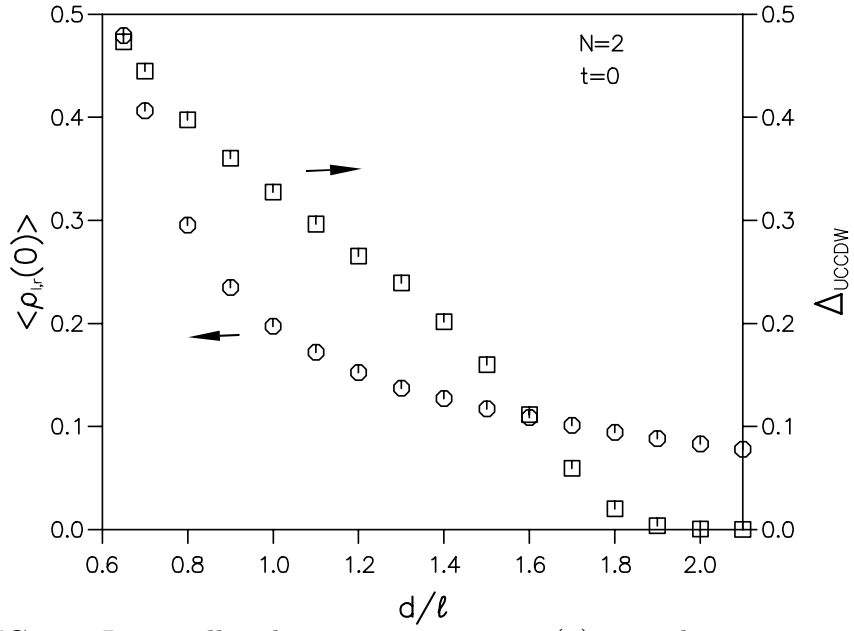

FIG. 6. Interwell order parameter $\left\langle\rho_{l, r}(0)\right\rangle$ and energy gap $\Delta_{U C C D W}$ as a function of the layer separation. These results corresponds to the case $N=2$ and $t=0$. 


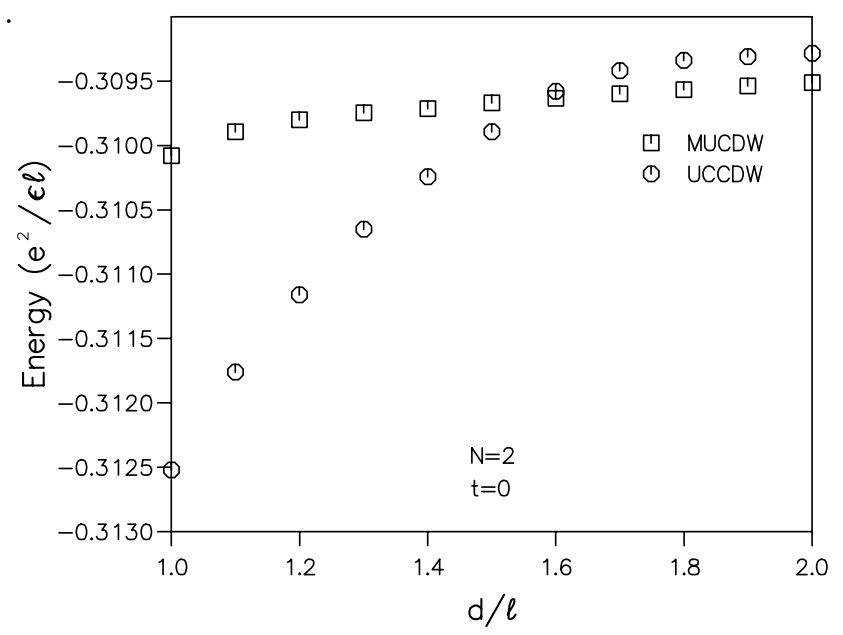

FIG. 7. Energy as a function of the layer separation, for the MUCDW and UCCDW phase. The results corresponds to $N=2$ and $t=0$.

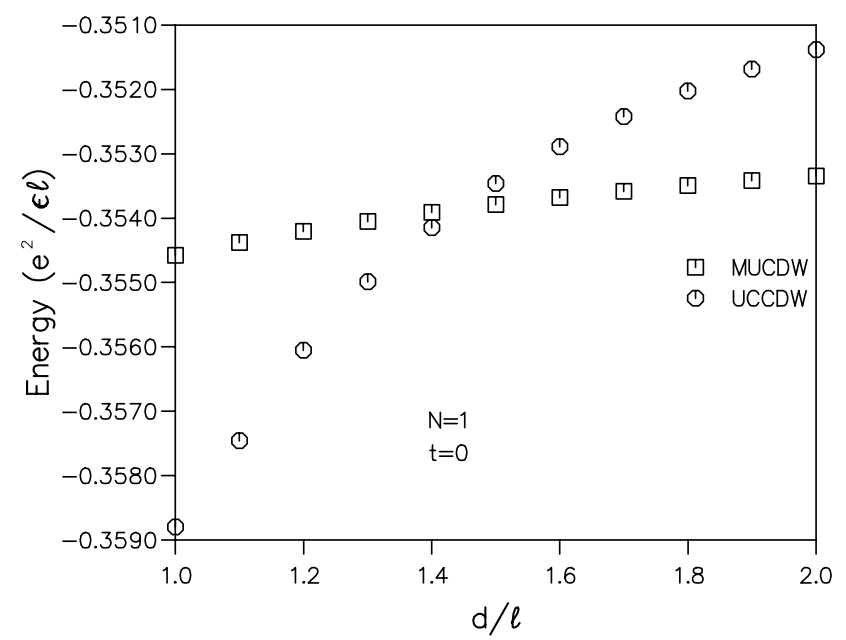

FIG. 8. Energy as a function of the layer separation, for the MUCDW and UCCDW phase. The results corresponds to $N=1$ and $t=0$. 